\title{
Necessary and Sufficient Conditions for Set-Valued Maps with Set Optimization
}

\author{
Abdessamad Oussarhan $(\mathbb{D}$ and Ikram Daidai \\ Faculté des Sciences et Techniques, Université Cadi Ayyad, BP 549, Marrakech, Morocco \\ Correspondence should be addressed to Abdessamad Oussarhan; abdessamad.oussa@gmail.com
}

Received 15 August 2017; Accepted 10 December 2017; Published 1 January 2018

Academic Editor: Patricia J. Y. Wong

Copyright (c) 2018 Abdessamad Oussarhan and Ikram Daidai. This is an open access article distributed under the Creative Commons Attribution License, which permits unrestricted use, distribution, and reproduction in any medium, provided the original work is properly cited.

Optimality conditions are studied for set-valued maps with set optimization. Necessary conditions are given in terms of $S$-derivative and contingent derivative. Sufficient conditions for the existence of solutions are shown for set-valued maps under generalized quasiconvexity assumptions.

\section{Introduction}

In recent years, a great attention has been paid to set-valued optimization problems; many authors (see, e.g., [1-7]) have concentrated on the problems with and without constraints:

$$
\begin{aligned}
\operatorname{minimize} & F(x), \\
& x \in M, \\
\operatorname{minimize} & F(x), \\
& G(x) \cap\left(-K_{Z}\right) \neq \emptyset, \\
& x \in M,
\end{aligned}
$$

where $F$ and $G$ are set-valued maps defined between two Banach spaces $X, Y$ and $X, Z$, respectively, $K_{Z}$ is the pointed closed convex cone of $Z$, and $M$ is a nonempty subset of $X$.

Studies on these problems consider two types of solutions: vector solution, given by a vector optimization, and set solution, given by a set optimization.

The vector solution cannot be often used in practice, since it depends only on special element of image set of solution and the other elements are ignored; therefore the solution concept in vector optimization is sometimes improper. In order to avoid this drawback, Kuroiwa [8] introduced in the first time the concept of set solution by using practically relevant order relations for sets. This leads to solution concepts for set-valued optimization problems based on comparisons among values of the set-valued objective map. Hernández et al. [9] gives some links between solutions concepts in vector and set optimization.

Taa [7] gives necessary and sufficient conditions for unconstraint vector optimization in terms of $S$-derivatives. Jahn and Khan [3] establish optimality conditions for unconstrained vector optimization under generalized convexity assumptions. Alonso-Durán and Rodríguez-Marín [10] give optimality conditions for the considered problems in set optimization using directional derivatives under pseudoconvexity assumptions and with the notion of the contingent derivative. In this paper we study necessary conditions for both problems in terms of $S$-derivatives with set optimization and we derive sufficient conditions under weaker notion of pseudoconvexity assumptions that are given in [3].

This paper is divided into three sections. In the first Section we collect some of the concepts required for the paper. Section 2 is devoted to the necessary optimality conditions for the unconstrained and the constrained set optimization and Section 3 deals with the sufficient optimality conditions in set optimization.

\section{Preliminaries}

Let $X, Y$, and $Z$ be real normed spaces, where $Y$ and $Z$ are partially ordered by convex pointed cones with nonempty 
interiors $K_{Y}$ and $K_{Z}$, respectively. $Y^{*}$ and $Z^{*}$ will denote the continuous duals of $X$ and $Y$, respectively. The collection of nonempty subsets of $Y$ will be denoted by $\wp(Y)$.

Let $F: X \rightrightarrows Y$ be a set-valued map. We recall that the effective domain and the graph of $F$ are defined by

$$
\begin{aligned}
\operatorname{dom}(F) & :=\{x \in X \mid F(x) \neq \emptyset\}, \\
\operatorname{Gr}(F) & :=\{(x, y) \in X \times Y \mid y \in F(x)\} .
\end{aligned}
$$

Let $G: X \rightrightarrows Z$ be a set-valued map and let us suppose that $\operatorname{dom} F=\operatorname{dom} G=M$ with $M \neq \emptyset$.

Research in set-valued optimization has concentrated on the problems with and without constraints:

$$
\begin{aligned}
\operatorname{minimize} & F(x) \\
& x \in M . \\
\operatorname{minimize} & F(x) \\
& G(x) \cap\left(-K_{Z}\right) \neq \emptyset, \\
& x \in M .
\end{aligned}
$$

A solution $\bar{x} \in M$ for these problems with the criterion of vector optimization is defined as a generalization of the notion established by Pareto. We recall this concept in the following definition. Let $F(M)=\bigcup_{x \in M} F(x)$.

Definition 1. Let $\bar{x} \in M$. It is said that $\bar{x}$ is

(i) a minimum solution for (3) and we denote $\bar{x} \epsilon$ $\min \left(F, K_{Y}\right)$ (or $\left.\bar{x} \in \min (F)\right)$, if there exists $\bar{y} \in F(\bar{x})$ such that

$$
(F(M)-\bar{y}) \cap\left(-K_{Y}\right)=\{0\} ;
$$

(ii) a weak minimum solution for (3) and we denote $\bar{x} \epsilon$ $\mathrm{W} \min \left(F, K_{Y}\right)($ or $\bar{x} \in \mathrm{W} \min (F))$, if there exists $\bar{y} \epsilon$ $F(\bar{x})$ such that

$$
(F(M)-\bar{y}) \cap\left(-\operatorname{int}\left(K_{Y}\right)\right)=\emptyset .
$$

Let $\leq^{l}\left(<^{l}\right.$, resp.) be the following relation defined between two nonempty subsets $A, B$ of $Y$ :

$$
\begin{aligned}
& A \leq^{l} B \Longleftrightarrow B \subset A+K_{Y}, \\
&\left(\text { resp. } A<B \Longleftrightarrow B \subset A+\operatorname{int}\left(K_{Y}\right)\right) .
\end{aligned}
$$

Using the above relations, Kuroiwa [8], in a natural way, introduced the following notion of l-minimal set (weakly lminimal set, resp.).

Definition 2. Let $\mathcal{S} \subset \wp(Y)$. It is said that $A \in \mathcal{S}$ is

(i) a lower minimal (or l-minimal) set of $\mathcal{S}$, if $B \in \mathcal{S}$ and $B \leq^{l} A$ imply $A \leq^{l} B$. The family of l-minimal sets of $\mathcal{S}$ is denoted by $1-\min \left(\mathcal{S}, K_{Y}\right)($ or $1-\min (\mathcal{S}))$; (ii) a lower weak minimal (or l-w minimal) set of $\mathcal{S}$, if $B \in \mathcal{S}$ and $B<^{l} A$ imply $A<^{l} B$. The family of weakly l-minimal sets of $\mathcal{S}$ is denoted by $1-\mathrm{W} \min \left(\mathcal{S}, K_{Y}\right)$ (or $1-\mathrm{W} \min (\mathcal{S})$ ).

In this way, the problems (3) and (4) can be written in set optimization with the following forms:

$$
\begin{array}{ll}
\text { 1-minimize } & F(x) \\
& x \in M . \\
\text { 1-minimize } & F(x) \\
& G(x) \cap\left(-K_{Z}\right) \neq \emptyset, \\
& x \in M .
\end{array}
$$

In these cases, $\bar{x}$ is a 1 -minimum (1- w minimum, resp.) solution of $F$, if $\bar{x} \in M$ (with $G(\bar{x}) \cap-K_{Z} \neq \emptyset$ in the problem $\left(\mathrm{SP}_{2}\right)$ ) and $F(\bar{x})$ is a l-minimal (l-w minimal, resp.) set of the family of images of $F$, that is, the family

$$
\mathscr{F}=\{F(x) \mid x \in M\} .
$$

The next proposition supplies a characterization of 1w minimum (see [10, Proposition 18]).

Proposition 3. $\bar{x} \in M$ is an l-w minimal solution of $\left(S P_{1}\right)$ if and only if for each $x \in M$ one of the following conditions is satisfied:

(i) $F(x)<^{l} F(\bar{x})$ and $F(\bar{x})<^{l} F(x)$.

(ii) There exists $\bar{y} \in F(\bar{x})$ such that $(F(x)-\bar{y}) \cap$ $\left(-\operatorname{int}\left(K_{Y}\right)\right)=\emptyset$.

Let us recall the following definition.

Definition 4. Let $(\bar{x}, \bar{y}) \in \operatorname{Gr}(F)$. The contingent derivative $\operatorname{DF}(\bar{x}, \bar{y})$ is the set-valued map from $X$ into $Y$ defined by $y \in$ $\operatorname{DF}(\bar{x}, \bar{y})(x)$ if there exist sequences $\left(t_{n}\right) \rightarrow 0^{+},\left(x_{n}, y_{n}\right) \rightarrow$ $(x, y)$ such that

$$
\bar{y}+t_{n} y_{n} \in F\left(\bar{x}+t_{n} x_{n}\right), \quad \forall n \in \mathbb{N}
$$

The following definition has been introduced by Shi [6]. It is an extension of the set-valued derivative in Definition 4.

Definition 5. Let $(\bar{x}, \bar{y}) \in \operatorname{Gr}(F)$. The $S$-derivative $\operatorname{SF}(\bar{x}, \bar{y})$ is the set-valued map from $X$ into $Y$ defined by $y \in S F(\bar{x}, \bar{y})(x)$ if there exist sequences $\left.\left(t_{n}\right) \subset\right] 0,+\infty\left[,\left(x_{n}, y_{n}\right) \rightarrow(x, y)\right.$ such that

$$
\begin{aligned}
t_{n} x_{n} & \longrightarrow 0, \\
\bar{y}+t_{n} y_{n} & \in F\left(\bar{x}+t_{n} x_{n}\right),
\end{aligned}
$$

$\forall n \in \mathbb{N}$.

Remark 6. Let $(\bar{x}, \bar{y}) \in \operatorname{Gr}(F)$. It is easy to see the following:

(i) The set-valued derivatives $\operatorname{DF}(\bar{x}, \bar{y})$ and $\operatorname{SF}(\bar{x}, \bar{y})$ are positively homogeneous with closed graphs. 
(ii) $(0,0) \in \operatorname{Gr}(D F(\bar{x}, \bar{y})) \subset \operatorname{Gr}(\operatorname{SF}(\bar{x}, \bar{y}))$.

(iii) $\operatorname{DF}(\bar{x}, \bar{y})=\operatorname{SF}(\bar{x}, \bar{y})$ whenever the graph of $F$ is convex in $X \times Y$.

For sufficient condition for $1-\mathrm{w}$ minimal solution of problems $\left(\mathrm{SP}_{1}\right)$ and $\left(\mathrm{SP}_{2}\right)$, we need certain convexity assumptions which are taken from $[3,4]$.

Definition 7. Let $\Gamma \subset Y$ and $(\bar{x}, \bar{y}) \in \operatorname{Gr}(F) . F$ is called $\Gamma$ contingently quasiconvex at $(\bar{x}, \bar{y})$, if, for every $x \in M$, the condition $(F(x)-\bar{y}) \cap \Gamma \neq \emptyset$ ensures that $D F(\bar{x}, \bar{y})(x-\bar{x}) \cap \Gamma \neq$ $\emptyset$.

Definition 8. We say that $F$ is $K_{Y}$-pseudoconvex at $(\bar{x}, \bar{y}) \in$ $\mathrm{Gr}(F)$ if and only if

$$
F(x)-\bar{y} \subset D F(\bar{x}, \bar{y})(x-\bar{x})+K_{Y} .
$$

Remark 9. Let $\Gamma \subset Y$. The $\Gamma$-contingent quasiconvexity reduces to the $K_{Y}$-pseudoconvexity.

\section{Necessary Optimality Conditions}

According to derived necessary condition, we recall the following notion of the strict l-w minimum and the concept of the $K_{Y}$-w minimal property given in [10].

Definition 10. Let $\bar{x}$ be an l-w minimum solution of $\left(\mathrm{SP}_{1}\right)$. $\bar{x}$ is called strict $1-\mathrm{w}$ minimum of $\left(\mathrm{SP}_{1}\right)$, if there exists a neighbourhood $U$ of $\bar{x}$ such that $F(x) \nless^{l} F(\bar{x})$ for all $x \in$ $U \cap M$.

Definition 11 (domination property). A subset $A \subset Y$ has the $K_{Y}$-W minimal property if for all $y \in A$ there exists $a \in \mathrm{W} \min (A)$ such that $a-y \in\left(-\operatorname{int}\left(K_{Y}\right)\right) \cup\{0\}$.

The following Lemma has been established in [10] without proof; we give a simple proof for reader's convenience.

Lemma 12. Let $x, \bar{x} \in M$ and $\bar{y} \in F(\bar{x})$. If $F(x) \nless^{l} F(\bar{x})$, $W \min (F(\bar{x}))=\{\bar{y}\}$, and $F(\bar{x})$ has the $K_{Y}-w$ minimal property, one has

$$
(F(x)-\bar{y}) \cap\left(-\operatorname{int}\left(K_{Y}\right)\right)=\emptyset .
$$

Proof. Suppose the contrary; then there exist $y \in F(x)$ and $k \in \operatorname{int}\left(K_{Y}\right)$ such that

$$
\bar{y}=y+k \in F(x)+\operatorname{int}\left(K_{Y}\right) .
$$

Since $\mathrm{W} \min (F(\bar{x}))=\{\bar{y}\}$ and $F(\bar{x})$ have the $K_{Y}$-W minimal property we get

$$
y_{1}-\bar{y} \in \operatorname{int}\left(K_{Y}\right) \cup\{0\} \quad \forall y_{1} \in F(\bar{x}),
$$

that is,

$$
y_{1} \in \bar{y}+\operatorname{int}\left(K_{Y}\right) \cup\{0\} \quad \forall y_{1} \in F(\bar{x}) .
$$

From (13) we have

$$
y_{1} \in F(x)+\operatorname{int}\left(K_{Y}\right), \quad \forall y_{1} \in F(\bar{x}),
$$

and hence

$$
F(\bar{x}) \subset F(x)+\operatorname{int}\left(K_{Y}\right), \quad\left(\text { i.e., } F(x)<^{l} F(\bar{x})\right) .
$$

This contradicts $F(x) \nless^{l} F(\bar{x})$.

Necessary conditions for the problem $\left(\mathrm{SP}_{1}\right)$ are given in the following.

Theorem 13. Let $\bar{x}$ be a strict l-w minimum of $\left(S P_{1}\right)$. If $W \min (F(\bar{x}))=\{\bar{y}\}$ and $F(\bar{x})$ has the $K_{Y}-w$ minimal property, then

$$
\operatorname{SF}(\bar{x}, \bar{y})(x) \cap\left(-\operatorname{int}\left(K_{Y}\right)\right)=\emptyset, \quad \forall x \in M .
$$

Proof. Suppose the contrary; then there exist $x \in M$ and $y \in$ $Y$ such that

$$
y \in \operatorname{SF}(\bar{x}, \bar{y})(x) \cap\left(-\operatorname{int}\left(K_{Y}\right)\right),
$$

and hence there exist $\left(t_{n}\right)_{n \in \mathbb{N}}>0$ and $\left(x_{n}, y_{n}\right) \rightarrow(x, y)$ such that

$$
\begin{aligned}
& t_{n} x_{n} \longrightarrow 0, \\
& t_{n} y_{n} \in F\left(\bar{x}+t_{n} x_{n}\right)-\bar{y},
\end{aligned}
$$

\section{$\forall n \in \mathbb{N}$,}

and from the hypothesis we have that $\bar{x}$ is a strict 1$\mathrm{w}$ minimum of $\left(\mathrm{SP}_{1}\right)$; then there exists a neighbourhood $U$ of $\bar{x}$ such that $F(x) \nless^{l} F(\bar{x})$ for all $x \in U \cap M$. Since $\bar{x}+t_{n} x_{n} \rightarrow \bar{x}$ then there exists $n_{0} \in \mathbb{N}$ such that $\bar{x}+t_{n} x_{n} \in U \cap M$ for all $n \geq n_{0}$ and then

$$
F\left(\bar{x}+t_{n} x_{n}\right) \nless^{l} F(\bar{x}), \quad \forall n \geq n_{0},
$$

by Lemma 12 and hypothesis we get

$$
\left[F\left(\bar{x}+t_{n} x_{n}\right)-\bar{y}\right] \cap\left(-\operatorname{int}\left(K_{Y}\right)\right)=\emptyset, \quad \forall n \geq n_{0} .
$$

On the other hand, $y \in-\operatorname{int}\left(K_{Y}\right)$; then there exists $n_{1} \in \mathbb{N}$ such that

$$
t_{n} y_{n} \in-\operatorname{int}\left(K_{Y}\right), \quad \forall n \geq n_{1}
$$

and for (20) we have

$$
\left[F\left(\bar{x}+t_{n} x_{n}\right)-\bar{y}\right] \cap\left(-\operatorname{int}\left(K_{Y}\right)\right) \neq \emptyset \quad \forall n \geq n_{1} .
$$

This contradicts (22) for all $n \geq \max \left(n_{0}, n_{1}\right)$.

As an immediate consequence we have the following corollary.

Corollary 14. Let $\bar{x}$ be an l-w minimum of $\left(S P_{1}\right)$. Let $\bar{y} \epsilon$ $F(\bar{x})$. Let us suppose that there exists a neighbourhood $U$ of $\bar{x}$ such that for each $x \in U \cap M$ one of the following conditions is satisfied:
(a) $\bar{y} \notin F(x)+\operatorname{int}\left(K_{Y}\right)$ or
(b) $\bar{y} \in W \min \left(F(x), K_{Y}\right)$. 
Then,

(i) $\bar{x}$ is a strict $l-w$ minimum of $\left(S P_{1}\right)$;

(ii) $S F(\bar{x}, \bar{y})(x) \cap\left(-\operatorname{int}\left(K_{Y}\right)\right)=\emptyset$, for all $x \in M$.

Proof. It is obvious that (b) $\Rightarrow$ (a) $\Rightarrow F(x) \nless^{l} F(\bar{x})$. Then if there exists a neighbourhood $U$ of $\bar{x}$ such that for each $x \in$ $U \cap M$ the condition (a) holds, we deduce from Definition 10 that (i) holds. On the other hand if (a) holds we have

$$
(F(x)-\bar{y}) \cap\left(-\operatorname{int}\left(K_{Y}\right)\right)=\emptyset, \quad \forall x \in U \cap M .
$$

By using similar arguments as in Theorem 13, we establish (ii).

Another consequences of Theorem 13 and Corollary 14 are given in the following corollaries.

Corollary 15. Let $\bar{x}$ be a strict l-w minimum of $\left(S P_{1}\right)$. If $F(x) \nless^{l} F(\bar{x}), W \min (F(\bar{x}))=\{\bar{y}\}$, and $F(\bar{x})$ has the $K_{Y^{-}}$ $w$ minimal property, then

$$
\operatorname{DF}(\bar{x}, \bar{y})(x) \cap\left(-\operatorname{int}\left(K_{Y}\right)\right)=\emptyset, \quad \forall x \in M .
$$

Corollary 16. Let $\bar{x}$ be an l-w minimum of $\left(S P_{1}\right)$. Let $\bar{y} \in F(\bar{x})$. Let us suppose that there exists a neighbourhood $U$ of $\bar{x}$ such that for each $x \in U \cap M$ one of the following conditions is satisfied:
(a) $\bar{y} \notin F(x)+\operatorname{int}\left(K_{Y}\right)$ or
(b) $\bar{y} \in W \min \left(F(x), K_{Y}\right)$.

Then

(i) $\bar{x}$ is a strict $l-w$ minimum of $\left(S P_{1}\right)$;

(ii) $\operatorname{DF}(\bar{x}, \bar{y})(x) \cap\left(-\operatorname{int}\left(K_{Y}\right)\right)=\emptyset$, for all $x \in M$.

Remark 17. If $\bar{x}$ is a strict l-w minimum solution of $\left(\mathrm{SP}_{1}\right)$ and $\bar{y} \in \mathrm{W} \min (F(\bar{x}))$, Theorem 13 and Corollaries 14 and 16 are not guaranteed if the other conditions are not satisfied. Indeed, let us recall the example considered in Alonso-Durán and Rodríguez-Marín [10]: let $F:(0,2) \rightrightarrows \mathbb{R}^{2}$ be defined by

$$
\begin{aligned}
& F(x) \\
& = \begin{cases}\left\{(y, z) \in \mathbb{R}^{2} \mid(y-2 x+2)^{2}+z^{2} \leq x^{2}\right\}, & \text { if } x \leq 1, \\
\left\{(y, z) \in \mathbb{R}^{2} \mid(y-2 x+2)^{2}+z^{2} \leq(2-x)^{2}\right\}, & \text { if } x>1 .\end{cases}
\end{aligned}
$$

Let $K_{Y}=\mathbb{R}_{+}^{2}$. Then $\bar{x}=1$ is a strict l-w minimum of $F$ and $\bar{y}=(-1,0) \in \mathrm{W} \min (F(1))$. But observe that $\mathrm{W} \min (F(\bar{x})) \neq$ $\{\bar{y}\}$ and for all neighbourhoods $U$ of $\bar{x}$ there exists $x \in U \cap M$ such $\bar{y} \in F(x)+\operatorname{int}\left(K_{Y}\right)$ and $\bar{y} \notin \mathrm{W} \min (F(x))$. On the other hand for all $k \in-\operatorname{int}\left(K_{Y}\right)$ and $x \in M$ we take $t_{n}=0$ for each $n \in \mathbb{N}$, then for every sequence $\left(x_{n}, y_{n}\right) \rightarrow(x, k)$ we have

$$
(-1,0)+t_{n} y_{n} \in F\left(1+t_{n} x_{n}\right), \quad \forall n \in \mathbb{N},
$$

that is

$$
D F(1,(-1,0))(x) \cap\left(-\operatorname{int}\left(K_{Y}\right)\right) \neq \emptyset, \quad \forall x \in M .
$$

Hence

$$
S F(1,(-1,0))(x) \cap\left(-\operatorname{int}\left(K_{Y}\right)\right) \neq \emptyset, \quad \forall x \in M .
$$

In the following we are going to prove necessary optimality conditions for $\left(\mathrm{SP}_{2}\right)$ in terms of contingent derivative.

In the sequel the couple $(F, G)$ is a set-valued map from $X$ into $Y \times Z$ defined by

$$
(F, G)(x)=(F(x), G(x)) .
$$

Let $\bar{z} \in G(\bar{x}) \cap\left(-K_{Z}\right)$ and we consider the following problem $\left(\mathrm{SP}_{3}\right)$ with respect to $K_{Y} \times\left(K_{Z}+\bar{z}\right)$ :

$$
\begin{array}{ll}
\text { l-minimize } & (F, G)(x), \\
\text { subject to } & x \in M
\end{array}
$$

The following result compares the set of strict 1$\mathrm{w}$ minimum solution of $\left(\mathrm{SP}_{2}\right)$ to the set of strict $\mathrm{l}-\mathrm{w}$ minimum solution of $\left(\mathrm{SP}_{3}\right)$.

Proposition 18. If $\bar{x}$ is a strict $l$-w minimal solution of $\left(\mathrm{SP}_{2}\right)$ then for all $\bar{z} \in G(\bar{x}) \cap\left(-K_{Z}\right), \bar{x}$ is a strict $l$-w minimal solution of $\left(\mathrm{SP}_{3}\right)$ with respect to $K_{Y} \times\left(K_{Z}+\bar{z}\right)$,

Proof. Suppose the contrary; then, for every neighbourhood $U$ of $\bar{x}$, there exists $x \in U \cap M$ such that

$$
(F, G)(\bar{x}) \subset(F, G)(x)+\operatorname{int}\left(K_{Y} \times K_{Z}+\bar{z}\right),
$$

then,

$$
\begin{aligned}
& F(\bar{x}) \subset F(x)+\operatorname{int}\left(K_{Y}\right), \\
& G(\bar{x}) \subset G(x)+\operatorname{int}\left(K_{Z}+\bar{z}\right),
\end{aligned}
$$

and since $\bar{z} \in G(\bar{x})$, we get

$$
\begin{gathered}
F(\bar{x}) \subset F(x)+\operatorname{int}\left(K_{Y}\right), \\
0 \in G(x)+K_{Z} .
\end{gathered}
$$

Thus for every neighbourhood $U$ of $\bar{x}$ there exists $x \in U \cap M$ such that

$$
\begin{aligned}
F(x) & <F(\bar{x}), \\
G(x) \cap\left(-K_{Z}\right) & \neq \emptyset .
\end{aligned}
$$

This contradicts $\bar{x}$ is a strict $1-\mathrm{w}$ minimal solution of $\left(\mathrm{SP}_{2}\right)$.

Let us formulate necessary conditions for the problem $\left(\mathrm{SP}_{2}\right)$. In the sequel we consider the following subset of $M$ :

$$
S=\left\{x \in M \mid G(x) \cap\left(-K_{Z}\right) \neq \emptyset\right\} .
$$

Theorem 19. Let $\bar{x}$ be a strict $l-w$ minimum solution of $\left(S P_{2}\right)$ and $\bar{z} \in G(\bar{x}) \cap\left(-K_{Z}\right)$. If $F(\bar{x})$ has the $K_{Y}-w$ minimal property and $W \min \left(F(\bar{x}), K_{Y}\right)=\{\bar{y}\}$, then

$$
\begin{aligned}
D(F, G)(\bar{x}, \bar{y}, \bar{z})(x) \cap\left(-\operatorname{int}\left(K_{Y} \times\left(K_{Z}+\bar{z}\right)\right)\right) & =\emptyset, \\
\forall x & \in M .
\end{aligned}
$$


Proof. Suppose the contrary; then there exist $x \in M$ and $(y, z) \in Y \times Z$ such that

$$
\begin{aligned}
(y, z) \in & D(F, G)(\bar{x}, \bar{y}, \bar{z})(x) \\
& \cap\left(-\operatorname{int}\left(K_{Y} \times\left(K_{Z}+\bar{z}\right)\right)\right),
\end{aligned}
$$

and hence there exist $\left(t_{n}\right)_{n \in \mathbb{N}} \rightarrow 0^{+}$and $\left(x_{n}, y_{n}, z_{n}\right) \rightarrow$ $(x, y, z)$ such that

$$
t_{n}\left(y_{n}, z_{n}\right) \in(F, G)\left(\bar{x}+t_{n} x_{n}\right)-(\bar{y}, \bar{z}), \quad \forall n \in \mathbb{N}
$$

On the other hand, $(y, z) \in-\operatorname{int}\left(K_{Y} \times\left(K_{Z}+\bar{z}\right)\right)$, then there exists $n_{1} \in \mathbb{N}$ such that

$$
\begin{aligned}
& t_{n} y_{n} \in-\operatorname{int}\left(K_{Y}\right), \\
& z_{n} \in-\operatorname{int}\left(K_{Z}+\bar{z}\right),
\end{aligned}
$$

$$
\forall n \geq n_{1}
$$

and hence

$$
\begin{aligned}
& t_{n} y_{n} \in-\operatorname{int}\left(K_{Y}\right), \\
& \bar{z}+t_{n} z_{n} \in-K_{Z}+\left(1-t_{n}\right) \bar{z}, \\
& \forall n \geq n_{1},
\end{aligned}
$$

as $t_{n} \rightarrow 0^{+}$there exists $n_{2} \in \mathbb{N}$ such that $1-t_{n}>0$ for every $n \geq n_{2}$, then $\left(1-t_{n}\right) \bar{z} \in-K_{Z}$. Let $N=\max \left(n_{1}, n_{2}\right)$, by (39) we get

$$
\begin{array}{r}
{\left[F\left(\bar{x}+t_{n} x_{n}\right)-\bar{y}\right] \cap\left(-\operatorname{int}\left(K_{Y}\right)\right) \neq \emptyset} \\
G\left(\bar{x}+t_{n} x_{n}\right) \cap\left(-K_{Z}\right) \neq \emptyset
\end{array}
$$

$$
\forall n \geq N
$$

From hypothesis we have $\bar{x}$ is a strict l-w minimum solution of $\left(\mathrm{SP}_{2}\right)$; then there exists a neighbourhood $U$ of $\bar{x}$ such that $F(x) \nless^{l} F(\bar{x})$ for all $x \in U \cap S$. Since $\bar{x}+t_{n} x_{n} \rightarrow \bar{x}$ then there exists $n_{0} \in \mathbb{N}$ such that $\bar{x}+t_{n} x_{n} \in U \cap M$ for all $n \geq n_{0}$; thus $\bar{x}+t_{n} x_{n} \in U \cap S$ for all $n \geq \max \left(n_{0}, N\right)$; hence,

$$
F\left(\bar{x}+t_{n} x_{n}\right) \nless^{l} F(\bar{x}) \quad \forall n \geq \max \left(n_{0}, N\right),
$$

and, by Lemma 12 and hypothesis, we get

$$
\begin{aligned}
{\left[F\left(\bar{x}+t_{n} x_{n}\right)-\bar{y}\right] \cap\left(-\operatorname{int}\left(K_{Y}\right)\right) } & \emptyset \\
& \forall n \geq \max \left(n_{0}, N\right) .
\end{aligned}
$$

This contradicts (42).

As an immediate consequence we have the following corollary.

Corollary 20. Let $\bar{x}$ be an l-w minimum of $\left(S P_{2}\right)$ and $\bar{z} \epsilon$ $G(\bar{x}) \cap\left(-K_{Z}\right)$. Let $\bar{y} \in F(\bar{x})$. Let us suppose that there exists a neighbourhood $U$ of $\bar{x}$ such that for each $x \in U \cap S$ one of the following conditions is satisfied:

(a) $\bar{y} \notin F(x)+\operatorname{int}\left(K_{Y}\right)$ or (b) $\bar{y} \in W \min \left(F(x), K_{Y}\right)$.

Then

(i) $\bar{x}$ is a strict l-w minimum of $\left(S P_{2}\right)$;

(ii) $D(F, G)(\bar{x}, \bar{y}, \bar{z})(x) \cap\left(-\operatorname{int}\left(K_{Y} \times\left(K_{Z}+\bar{z}\right)\right)\right)=$ $\emptyset$, for all $x \in M$.

\section{Sufficient Optimality Conditions}

It is well known from vector optimization that we can derive sufficient condition under $\Gamma$-contingently quasiconvex assumptions. Next, we establish sufficient condition with similar assumptions for set optimization. The following terminology is used. Let $Y^{*}$ denote the dual space of $Y$, and let

$$
K_{Y}^{+}=\left\{u \in Y^{*} \mid u(y) \geq 0, \forall y \in K_{Y}\right\}
$$

denote the nonnegative dual cone of $K_{Y}$.

The next theorem provides a sufficient condition for the l-w minimum solution of $\left(\mathrm{SP}_{2}\right)$.

Theorem 21. Let $(\bar{x}, \bar{y}, \bar{z}) \in G r(F, G)$ and $M-\bar{x} \quad \mathrm{C}$ $\operatorname{dom}[D(F, G)(\bar{x}, \bar{y}, \bar{z})]$. Assume that there are $u \in K_{Y}^{+} \backslash\left\{0_{Y^{*}}\right\}$ and $v \in K_{Z}^{+}$such that $v(\bar{z})=0$ and

$$
u(y)+v(z) \geq 0
$$$$
\text { for every }(y, z) \in D\left(F+K_{Y}, G+K_{Z}\right)(\bar{x}, \bar{y}, \bar{z})(x-\bar{x}) \quad \forall x \in M
$$

If $\left(F+K_{Y}, G+K_{Z}\right): \widehat{M} \rightrightarrows Y \times Z$ is $\Gamma$-contingently quasiconvex at $(\bar{x}, \bar{y}, \bar{z})$ with

$$
\begin{aligned}
& \widehat{M}=\left\{x \in M \mid G(x) \cap\left(-K_{Z}+\text { cone }(\bar{z})-\text { cone }(\bar{z})\right)\right. \\
& \neq \emptyset\} \text {, } \\
& \Gamma=\left(-\operatorname{int}\left(K_{Y}\right)\right) \times\left(-K_{Z}+\text { cone }(\bar{z})-\text { cone }(\bar{z})\right),
\end{aligned}
$$

then $\bar{x}$ is an l-w minimal solution of $\left(S P_{2}\right)$ on $\widehat{M}$.

Proof. Let us show that, for every $x \in \widehat{M}$,

$$
D\left(F+K_{Y}, G+K_{Z}\right)(\bar{x}, \bar{y}, \bar{z})(x-\bar{x}) \cap \Gamma=\emptyset .
$$

Assume the contrary; then there exist $x^{\prime} \in \widehat{M}$ and $\left(y^{\prime}, z^{\prime}\right) \epsilon$ $Y \times Z$ such that

$$
\begin{aligned}
\left(y^{\prime}, z^{\prime}\right) & \in D\left(F+K_{Y}, G+K_{Z}\right)(\bar{x}, \bar{y}, \bar{z})\left(x^{\prime}-\bar{x}\right), \\
y^{\prime} & \in-\operatorname{int}\left(K_{Y}\right), \\
z^{\prime} & \in-K_{Z}+\operatorname{cone}(\bar{z})-\operatorname{cone}(\bar{z}) .
\end{aligned}
$$

Since $u \in K_{Y}^{+} \backslash\left\{0_{Y^{*}}\right\}, v \in K_{Z}^{+}$, and $v(\bar{z})=0$, we have

$$
u\left(y^{\prime}\right)+v\left(z^{\prime}\right)<0
$$

This contradicts (46). 
On the other hand, we have that $\left(F+K_{Y}, G+K_{Z}\right)$ is $\Gamma$ contingently quasiconvex at $(\bar{x}, \bar{y}, \bar{z})$; thus (48) ensures that there is no $x \in \widehat{M}$ such that

$$
\left(F(x)+K_{Y}, G(x)+K_{Z}\right)-(\bar{y}, \bar{z}) \cap \Gamma \neq \emptyset,
$$

that is

$$
\begin{array}{r}
\left(F(x)+K_{Y}-\bar{y}\right) \cap\left(-\operatorname{int}\left(K_{Y}\right)\right) \neq \emptyset \\
\left(G(x)+K_{Z}-\bar{z}\right) \cap\left(-K_{Z}+\operatorname{cone}(\bar{z})-\operatorname{cone}(\bar{z})\right) \neq \emptyset .
\end{array}
$$

Hence for every $x \in \widehat{M}$ there exists $\bar{y} \in F(\bar{x})$ such that

$$
(F(x)-\bar{y}) \cap\left(-\operatorname{int}\left(K_{Y}\right)\right)=\emptyset,
$$

by Proposition 3, and we deduce that $\bar{x}$ is a l-w minimal solution of $\left(\mathrm{SP}_{2}\right)$ on $\widehat{M}$.

As an immediate consequence we have the following corollary.

Corollary 22. Under the setting of Theorem 21, if the map $(F, G): \widehat{M} \rightrightarrows Y \times Z$ is $K_{Y} \times K_{Z}$-pseudoconvex at $(\bar{x}, \bar{y}, \bar{z})$ then $\bar{x}$ is a l-w minimal solution of $\left(S P_{2}\right)$ on $\widehat{M}$.

From Theorem 21, we obtain the following sufficient optimality condition for $\left(\mathrm{SP}_{1}\right)$.

Theorem 23. Let $(\bar{x}, \bar{y}) \in G r(F)$ and $M-\bar{x} \subset \operatorname{dom}[D F(\bar{x}, \bar{y})]$. Assume that there exists $u \in K_{Y}^{+} \backslash\left\{0_{Y^{*}}\right\}$ such that

$$
\begin{aligned}
& u(y) \geq 0 \\
& \text { for every } y \in D\left(F+K_{Y}\right)(\bar{x}, \bar{y})(x-\bar{x}) \quad \forall x \in M
\end{aligned}
$$

If $F+K_{Y}: M \rightrightarrows Y$ is $\Gamma$-contingently quasiconvex at $(\bar{x}, \bar{y})$ with, $\Gamma=-\operatorname{int}\left(K_{Y}\right)$, then $\bar{x}$ is an l-w minimal solution of $\left(S P_{1}\right)$.

The following two corollaries of the above result are immediate.

Corollary 24. Let $(\bar{x}, \bar{y}) \in G r(F)$ and $M-\bar{x} \quad C$ $\operatorname{dom}[\operatorname{DF}(\bar{x}, \bar{y})]$. Assume that

$$
\begin{aligned}
& D\left(F+K_{Y}\right)(\bar{x}, \bar{y})(x-\bar{x}) \cap\left(-\operatorname{int}\left(K_{Y}\right)\right)=\emptyset \\
& \forall x \in M .
\end{aligned}
$$

If $F+K_{Y}: M \rightrightarrows Y$ is $\Gamma$-contingently quasiconvex at $(\bar{x}, \bar{y})$ with, $\Gamma=-\operatorname{int}\left(K_{Y}\right)$, then $\bar{x}$ is an l-w minimal solution of $\left(S P_{1}\right)$.

Corollary 25. Let $(\bar{x}, \bar{y}) \in G r(F)$ and $M-\bar{x} \subset$ $\operatorname{dom}[\operatorname{DF}(\bar{x}, \bar{y})]$. Assume that

$$
D\left(F+K_{Y}\right)(\bar{x}, \bar{y})(x-\bar{x}) \cap\left(-\operatorname{int}\left(K_{Y}\right)\right)=\emptyset,
$$

$\forall x \in M$.

If $F: M \rightrightarrows Y$ is $K_{Y}$-pseudoconvex at $(\bar{x}, \bar{y})$, then $\bar{x}$ is an $l$ $w$ minimal solution of $\left(S P_{1}\right)$.

\section{Conclusions}

This paper deals with a set-valued optimization problem which involves a set-valued objective and set-valued constraints. Since such problems involve set-valued maps, optimality conditions are often given using various notions of setvalued derivatives. In this paper, we use the notion of the socalled $S$-derivative (and also the contingent derivative) to give necessary optimality conditions for the considered problems. For the sufficient optimality conditions, certain generalized notion of convexity is employed.

\section{Conflicts of Interest}

The authors declare that there are no conflicts of interest regarding the publication of this paper.

\section{References}

[1] T. Amahroq and A. Taa, "Sufficient conditions of optimality for multiobjective optimization problems with $\gamma$-paraconvex data," Studia Mathematica, vol. 124, no. 3, pp. 139-247, 1997.

[2] H. W. Corley, "Optimality conditions for maximizations of setvalued functions," Journal of Optimization Theory and Applications, vol. 58, no. 1, pp. 1-10, 1988.

[3] J. Jahn and A. A. Khan, "Generalized contingent epiderivatives in set-valued optimization: optimality conditions," Numerical Functional Analysis and Optimization, vol. 23, no. 7-8, pp. 807831, 2002.

[4] A. A. Khan, C. Tammer, and C. Zalinescu, Set-valued optimization. Vector optimization, Springer, Heidelberg, Germany, 2015.

[5] D. T. Luc, "Contingent derivatives of set-valued maps and applications to vector optimization," Programs in Mathematics, vol. 50, pp. 99-111, 1991.

[6] D. S. Shi, "Contingent derivative of the perturbation map in multiobjective optimization," Journal of Optimization Theory and Applications, vol. 70, no. 2, pp. 385-396, 1991.

[7] A. Taa, "Necessary and sufficient conditions for multiobjective optimization problems," Optimization. A Journal of Mathematical Programming and Operations Research, vol. 36, no. 2, pp. 97-104, 1996.

[8] D. Kuroiwa, "Some duality theorems of set-valued optimization with natural criteria," in Proceedings of the International Conference on Nonlinear Analysis and Convex Analysis, pp. 221-228, 1999.

[9] E. Hernández, L. Rodríguez-Marín, and M. Sama, "On solutions of set-valued optimization problems," Computers \& Mathematics with Applications, vol. 60, pp. 1401-1408, 2010.

[10] M. Alonso-Durán and L. Rodríguez-Marín, "Optimality conditions for set-valued maps with set optimization," Nonlinear Analysis, vol. 70, pp. 3057-3064, 2009. 


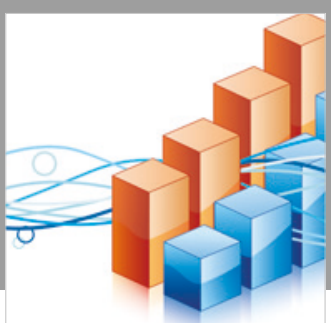

Advances in

Operations Research

\section{-n-m}
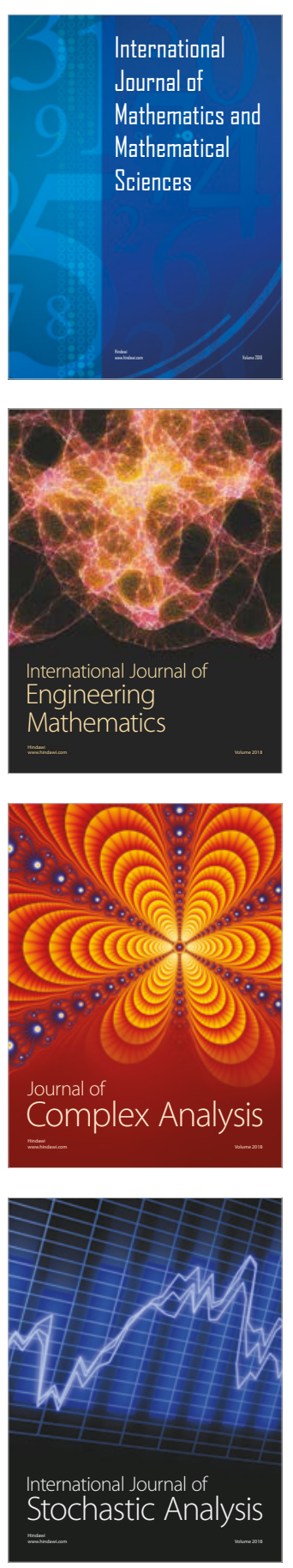
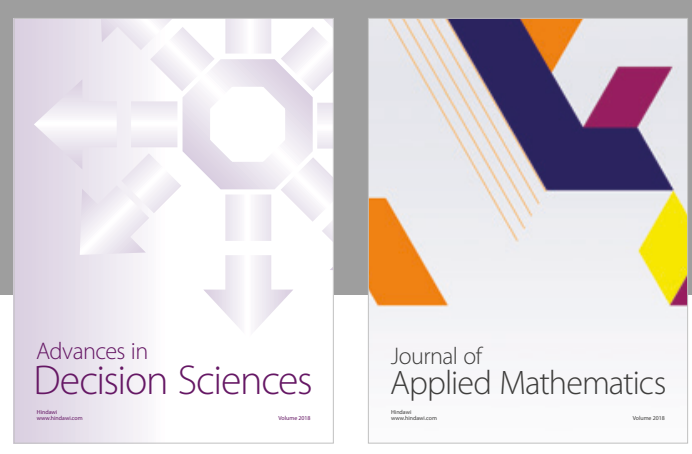

Journal of

Applied Mathematics
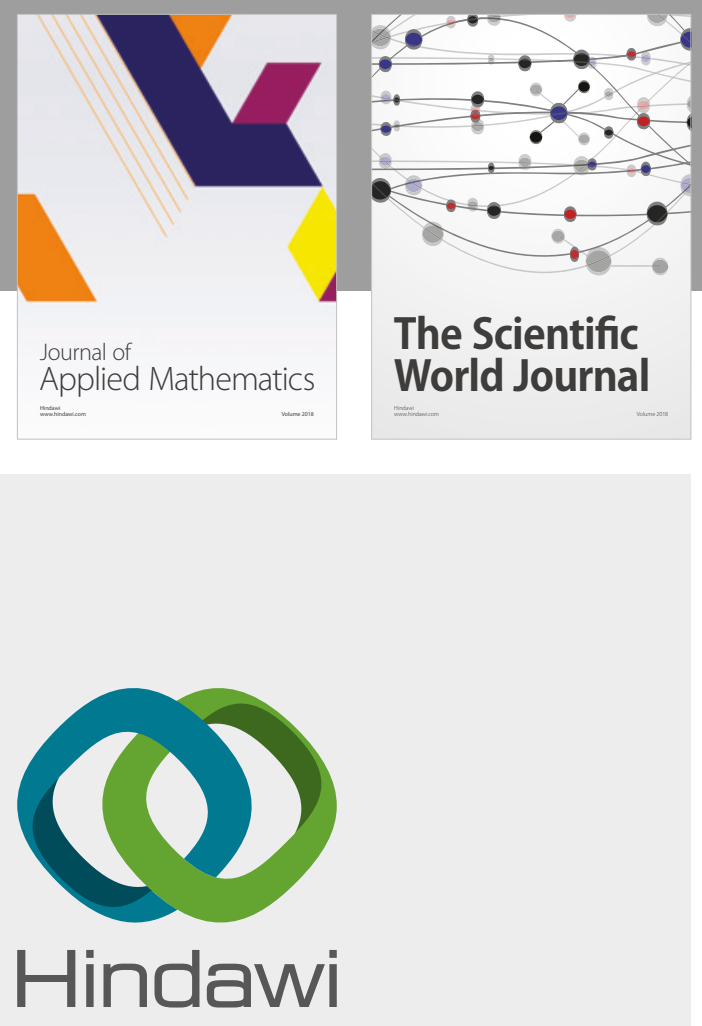

Submit your manuscripts at

www.hindawi.com

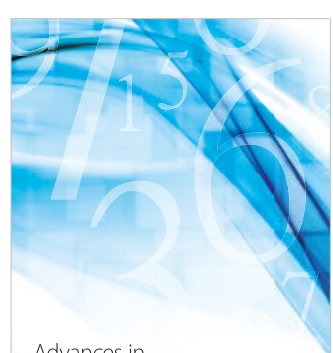

Advances in
Numerical Analysis
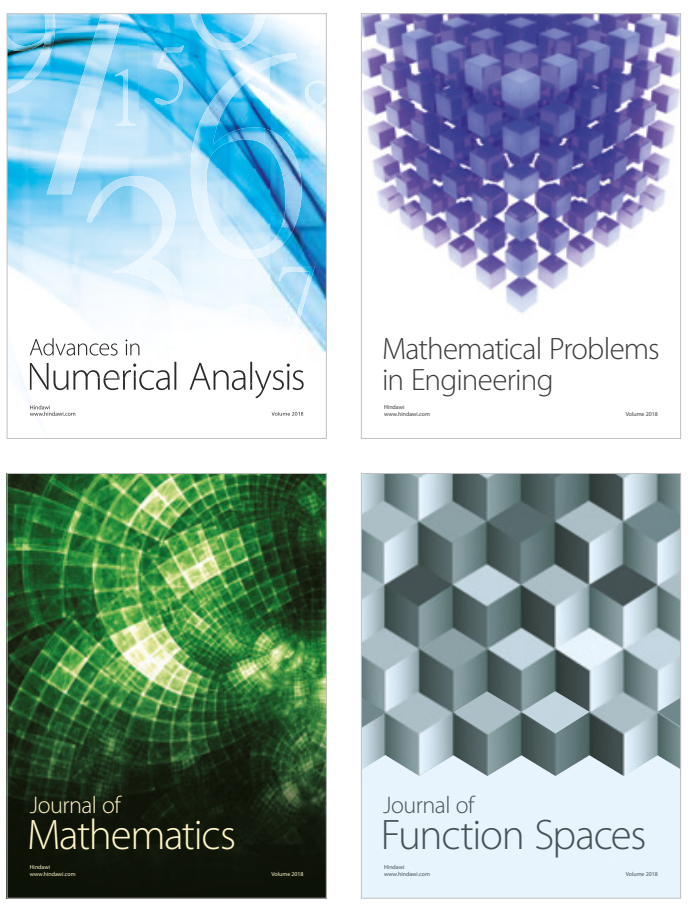

Mathematical Problems in Engineering

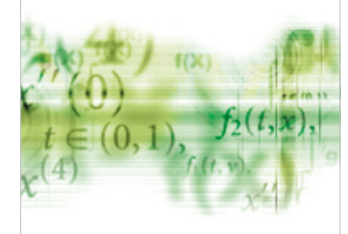

International Journal of

Differential Equations

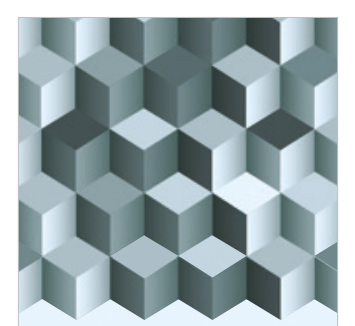

Journal of

Function Spaces

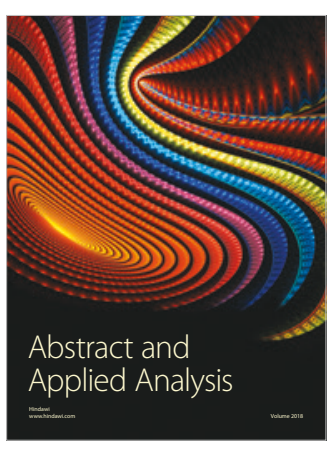

The Scientific

World Journal

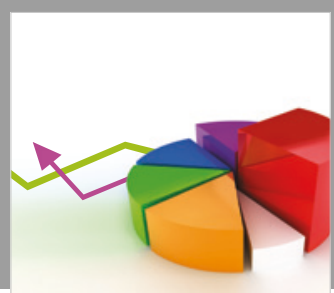

Journal of

Probability and Statistics
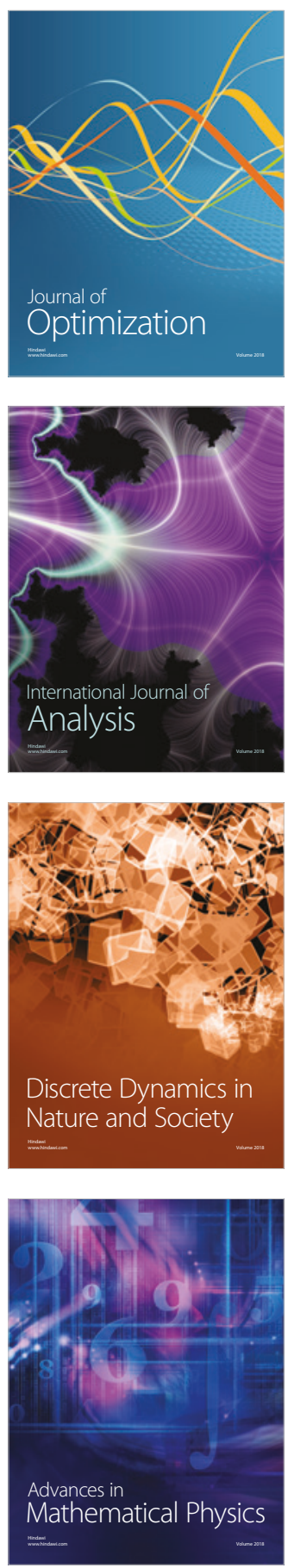